\title{
SIMULTANEOUS CODED PLANE WAVE IMAGING IN ULTRASOUND: PROBLEM FORMULATION AND CONSTRAINTS
}

Denis BUJOREANU

Denis FRIBOULET

Barbara NICOLAS

Hervé LIEBGOTT
CREATIS; CNRS (UMR 5220); INSERM (U1206); INSA Lyon; Université de Lyon, France. 


\section{Context - Motivation}

Ultrasound is often used to image fast transient events

10

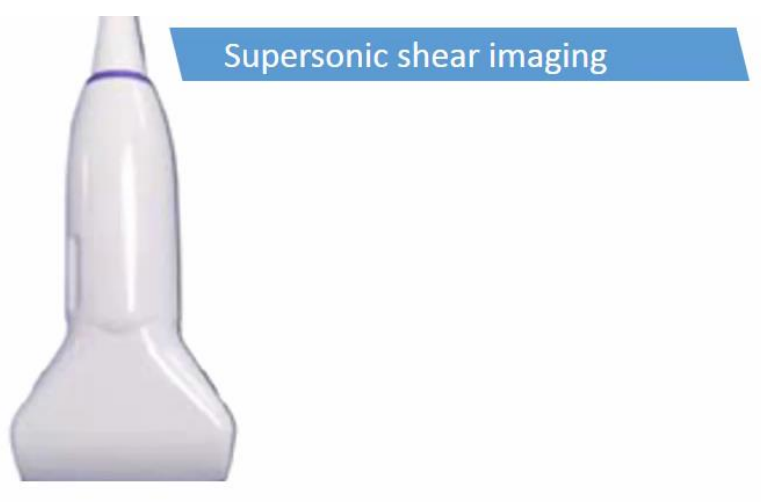

Elastography (@: www.institut-langevin.espci.fr)

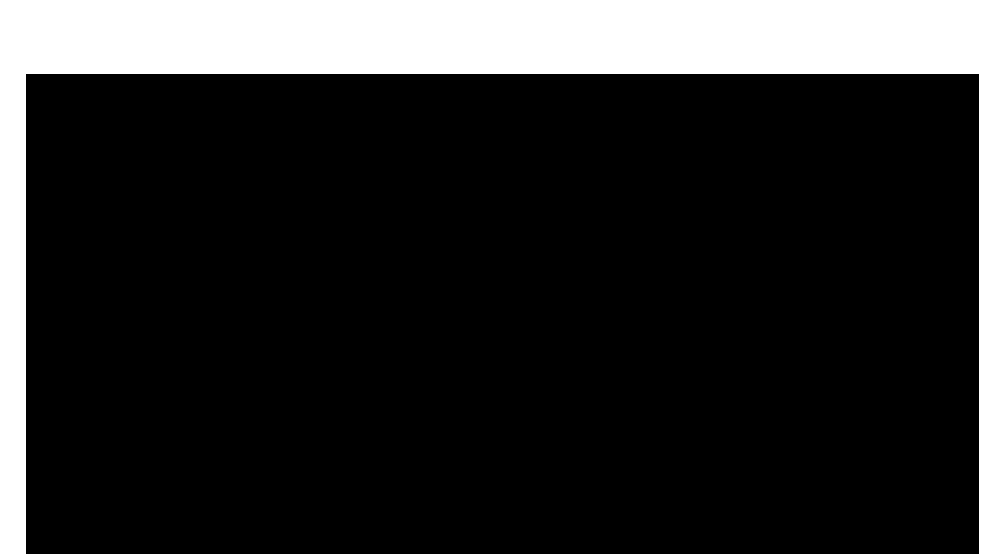

Flow imaging (Courtesy of Alfred Yu, University Waterloo, Canada)

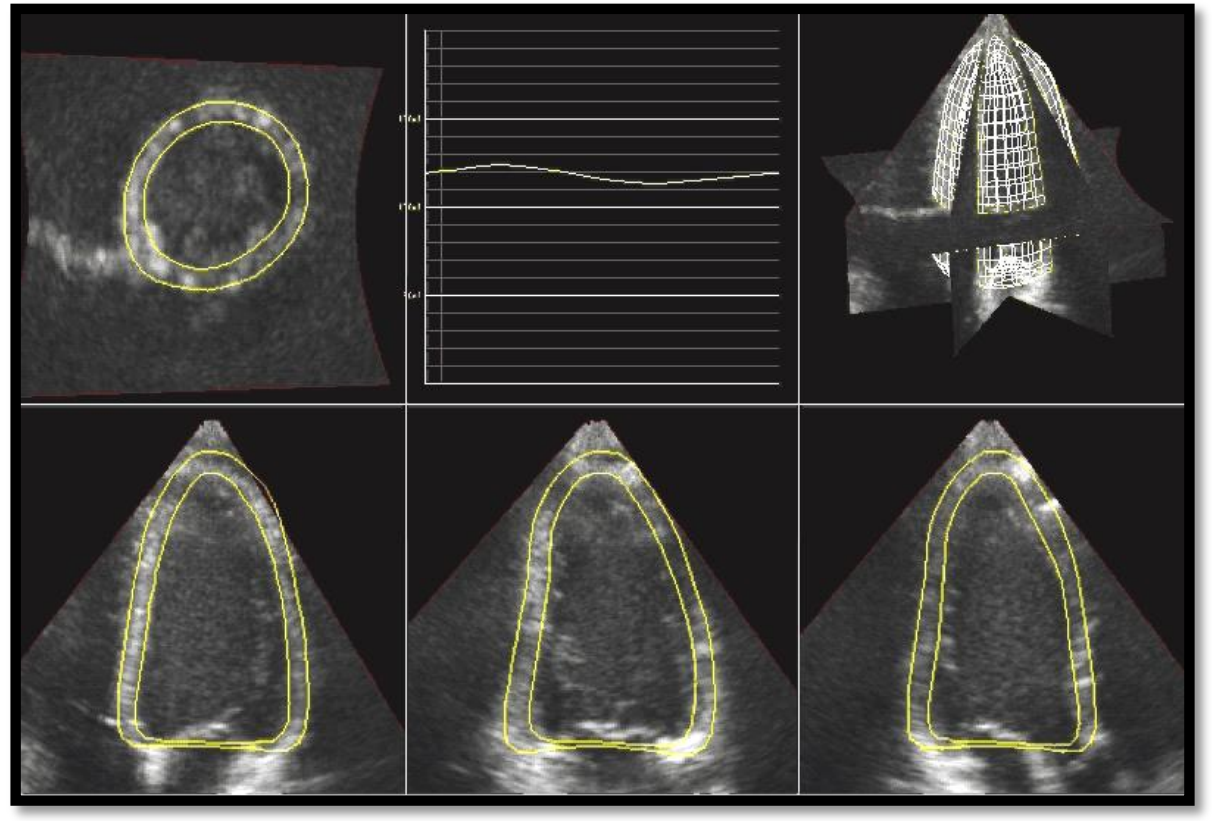

Strain imaging (Courtesy of Lasse Lovstakken NTNU Trrondheim)

High frame rate ultrasound could improve such techniques in $2 \mathrm{D}_{[1][2]}$ Higher acquisition rate of 2D frames $=>$ Higher frame rate of 3D frames

[1] Hansen, H. H. G., Saris, A. E. C. M., Vaka, N. R., Nillesen, M. M., \& de Korte, C. L. (2014). Ultrafast vascular strain compounding using plane wave transmission. Journal of biomechanics, 47(4), 815-823. [2] Vappou, J., Luo, J., \& Konofagou, E. E. (2010). Pulse wave imaging for noninvasive and quantitative measurement of arterial stiffness in vivo. American journal of hypertension, 23(4), 393-398 


\section{Plane wave imaging ${ }_{[1]}$}

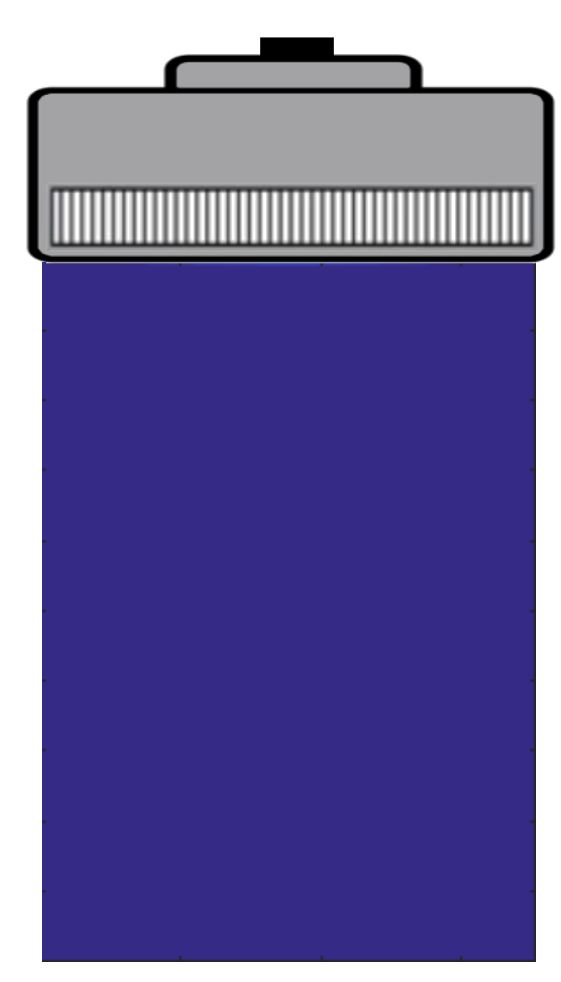

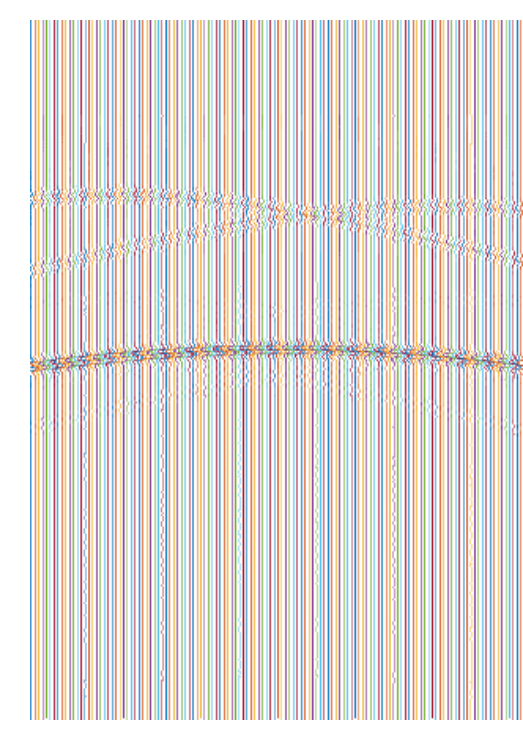

Received signals

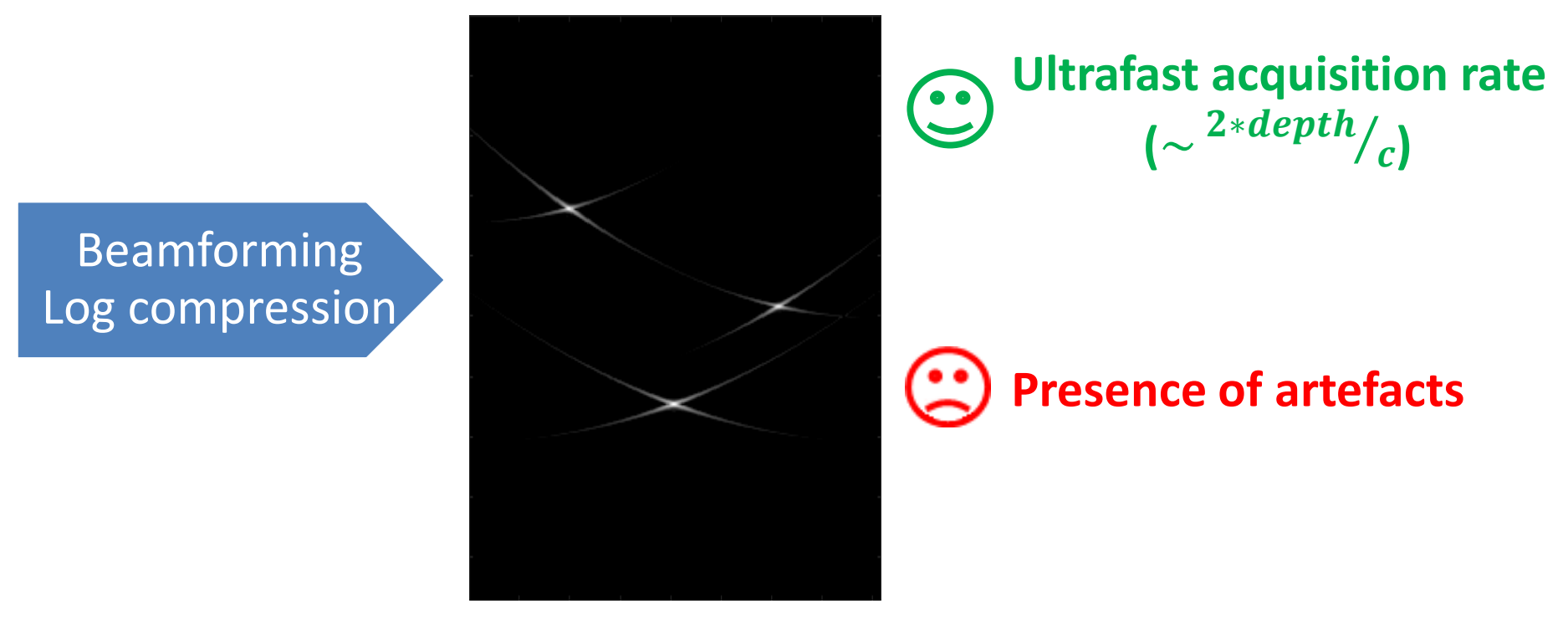

Final Image 


\section{Plane wave coherent compounding imaging ${ }_{[1]}$}

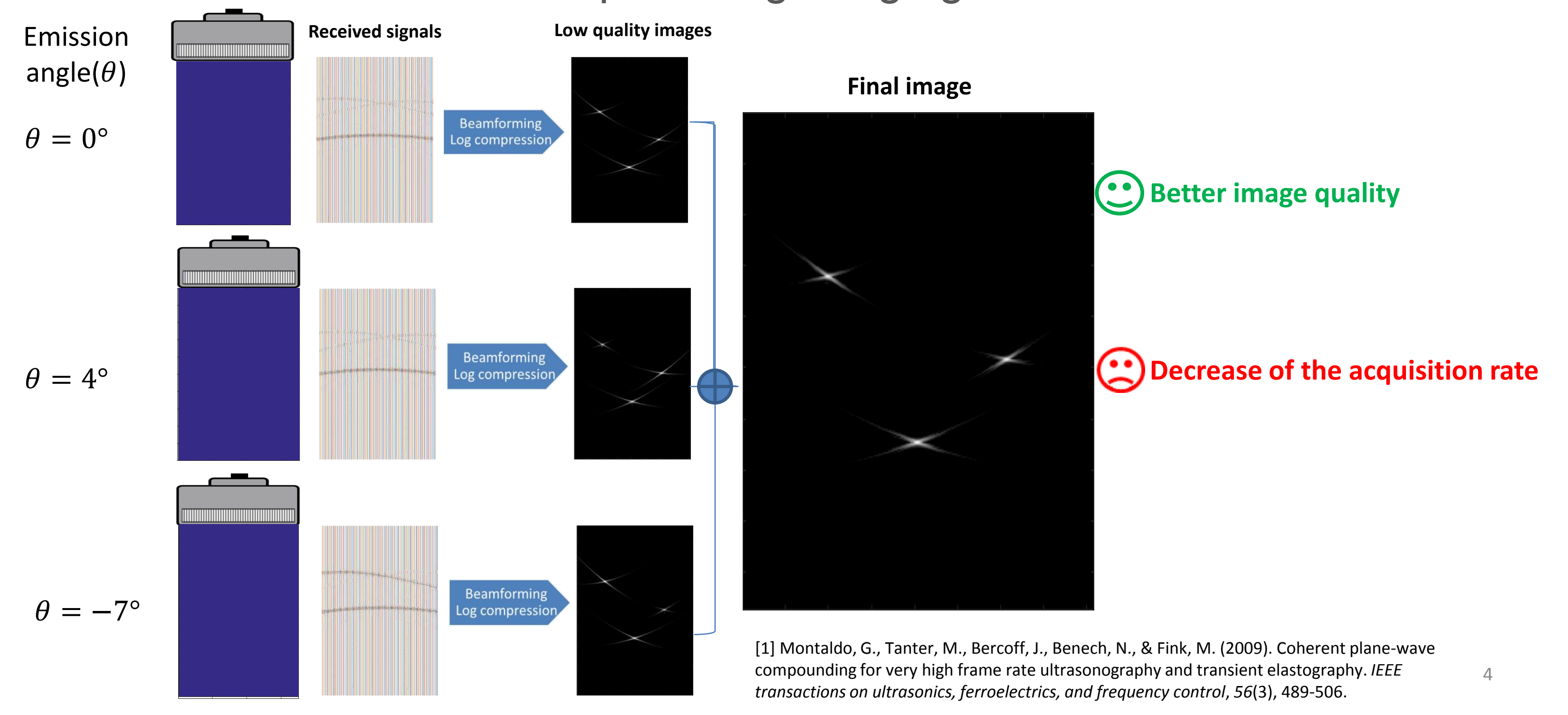




\section{Our proposal: Simultaneous emission of the plane waves}
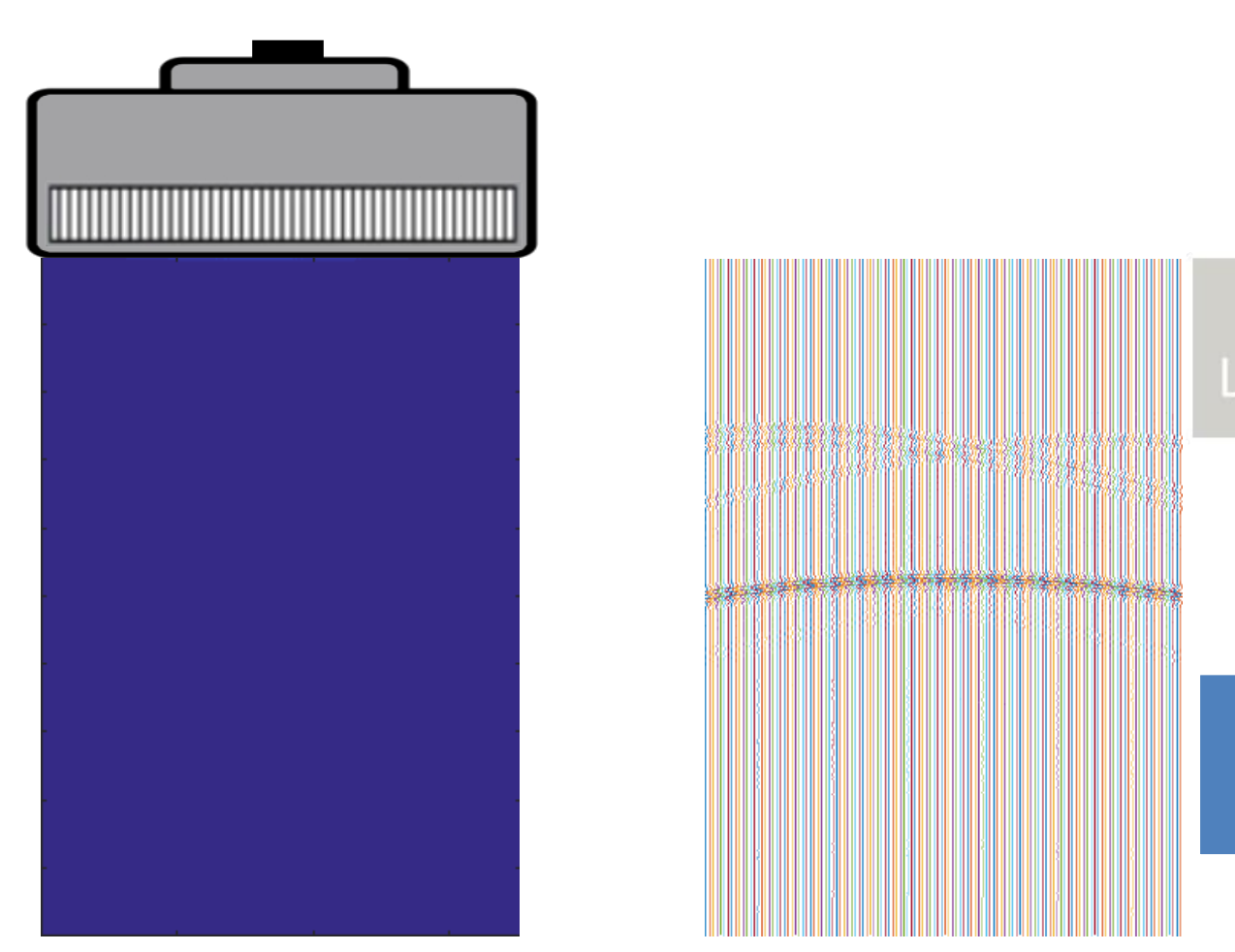

Inverse problem

approach

$\mathrm{Q}:$ What is the mathematical model of the system?

Q: What inverse problem approach to use in order to solve the model?

$\mathrm{Q}$ : What excitation signals to use?

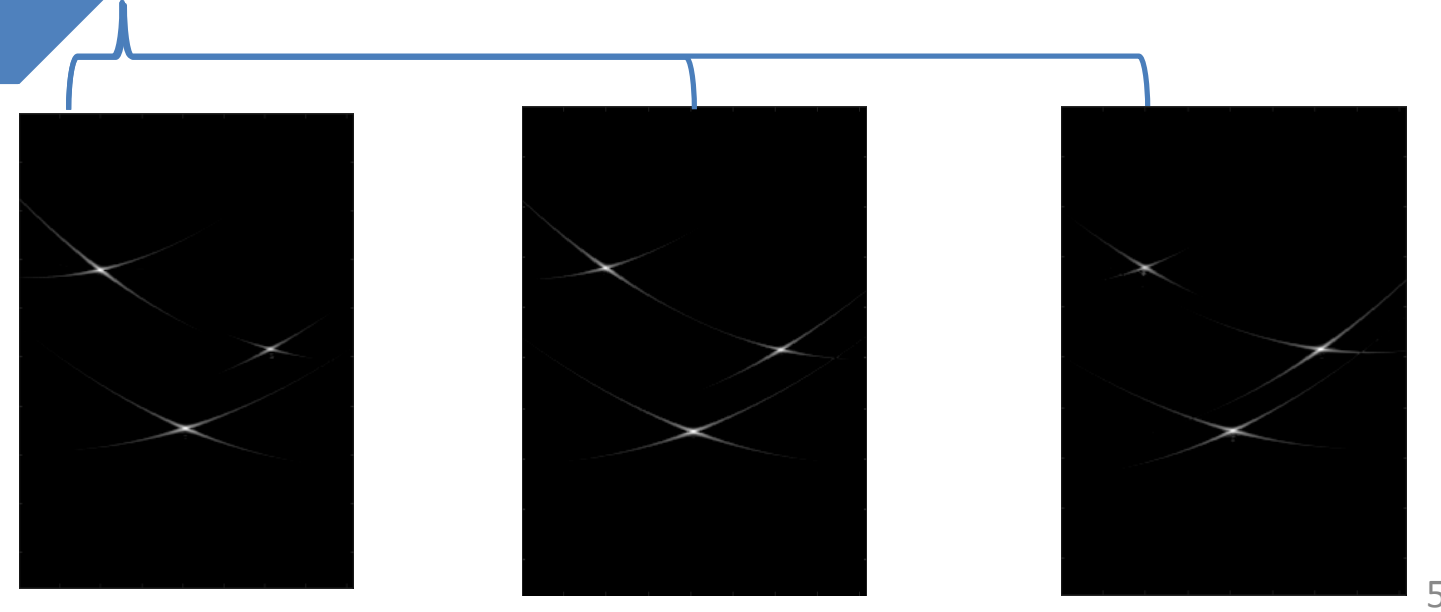

Direct beanforming $=>$ artefacis 


\section{At the end of this presentation you will know:}

The mathematical model of our system

The estimator that we use to solve our system

The conditions under which our system becomes well-posed

The excitation signals that allow the system to be well-conditioned - Codes 
Emission/reception of a plane wave carrying an arbitrary signal a(t)

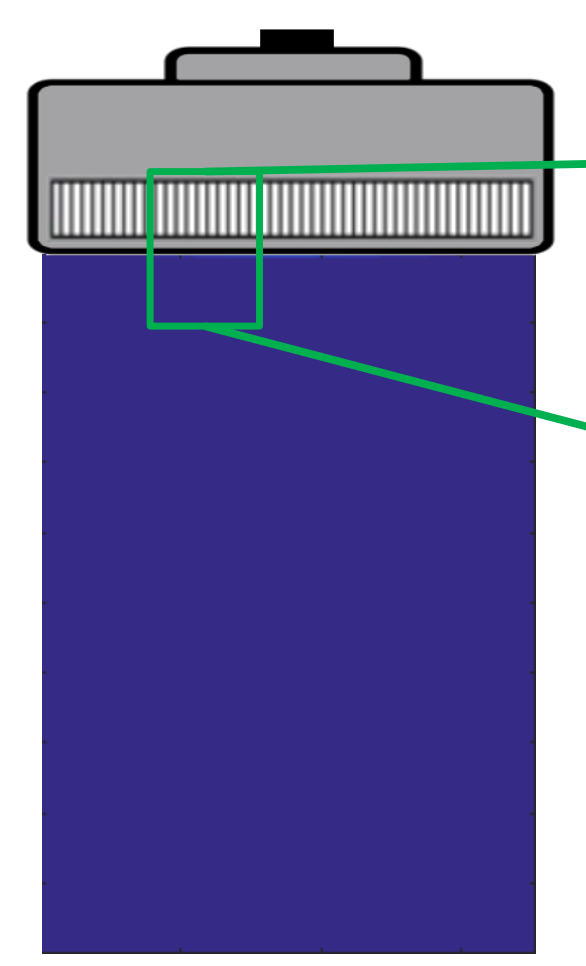

\section{jth Emitted signal:}

$$
\begin{array}{r}
a_{j}(t)=a(t) * \delta\left(t-t_{j}\right) \\
a(t)-\text { signal carried by the plane wave } \\
t_{j}=(j-1) \times \text { pitch } \times \tan (\theta) \\
\quad \theta-\text { tilt of the plane's wave wavefront }
\end{array}
$$

$t_{j}<t_{j+1}, \forall j \in\left[1, \ldots, N_{e}\right], \theta=4^{\circ}$

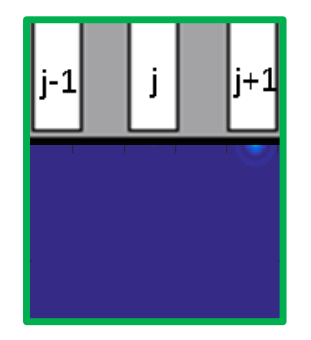

$t_{j}>t_{j+1}, \forall j \in\left[1, \ldots, N_{e}\right], \theta=-7^{\circ}$ 
Emission/reception of a plane wave carrying an arbitrary signal a(t)

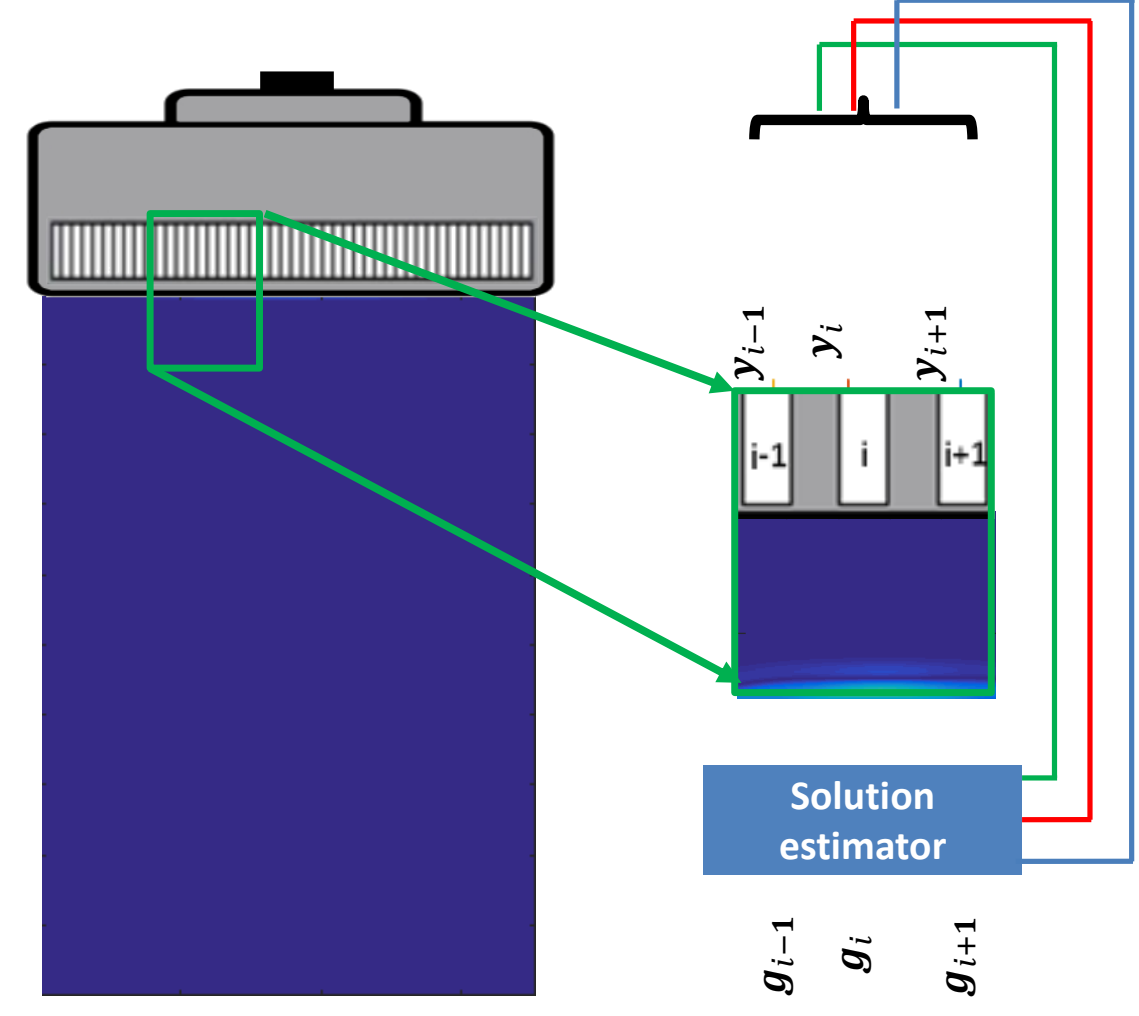

Beamformer

\section{ith Received signal:}

$y_{i}(t)=\sum_{j=1}^{N_{e}} a_{j}(t) * h_{j e}(t) * g_{j i}(t) * h_{i r}(t)+v_{i}(t)$

$h_{j e}(t), h_{i r}(t)$ - acousto-electrical impulse responses of elements at emission, reception

$g_{j i}(t)$ - impulse response of the medium when element $j$ emits and $i$ receives

$$
\begin{aligned}
& y_{i}(t)=a(t) * g_{i}(t)+v_{i}(t) \\
& g_{i}(t)=\sum_{j=1}^{N_{e}} \delta\left(t-t_{j}\right) * h_{j e}(t) * g_{j i}(t) * h_{i r}(t)
\end{aligned}
$$

$g_{i}(t)$ is the pulsed plane wave response of the medium seen by the $i$ th element of the probe

$g_{i}(t)$ - raw signals to be beamformed into the image of the medium 


\section{One plane wave model discretization}

$y_{i}(t)=a(t) * g_{i}(t)+v_{i}(t) \stackrel{\times \Perp \Perp \Perp 1}{\longrightarrow} \mathbf{y}_{i}=\mathbf{A} \times \mathbf{g}_{i}+\mathbf{v}_{i}$

$\mathbf{y}_{i}=\left[\begin{array}{llllll}y_{i}[0] & y_{i}[1] & y_{i}[2] & y_{i}[3] & \cdots & y_{i}\left[N_{y}\right.\end{array}\right]^{T}$

$\mathbf{g}_{i}=\left[\begin{array}{llllll}g_{i}[0] & g_{i}[1] & g_{i}[2] & g_{i}[3] & \cdots & \left.g_{i}\left[N_{g}\right]\right]^{T}\end{array}\right.$

$\mathbf{v}_{i}=\left[\begin{array}{llllll}v_{i}[0] & v_{i}[1] & v_{i}[2] & v_{i}[3] & \cdots & \left.v_{i}\left[N_{y}\right]\right]^{T}\end{array}\right.$

$\mathbf{A}=\left[\begin{array}{cccccccccc}a_{N_{a}-1} & a_{N_{a}-2} & \cdots & a_{0} & 0 & \cdots & 0 & 0 & \cdots & 0 \\ 0 & a_{N_{a}-1} & \cdots & a_{1} & a_{0} & \cdots & 0 & 0 & \cdots & 0 \\ \vdots & \vdots & \ddots & \vdots & \vdots & \ddots & \vdots & \vdots & \ddots & \vdots \\ 0 & 0 & \cdots & 0 & 0 & \cdots & a_{N_{a}-1} & a_{N_{a}-2} & \cdots & a_{0}\end{array}\right]$

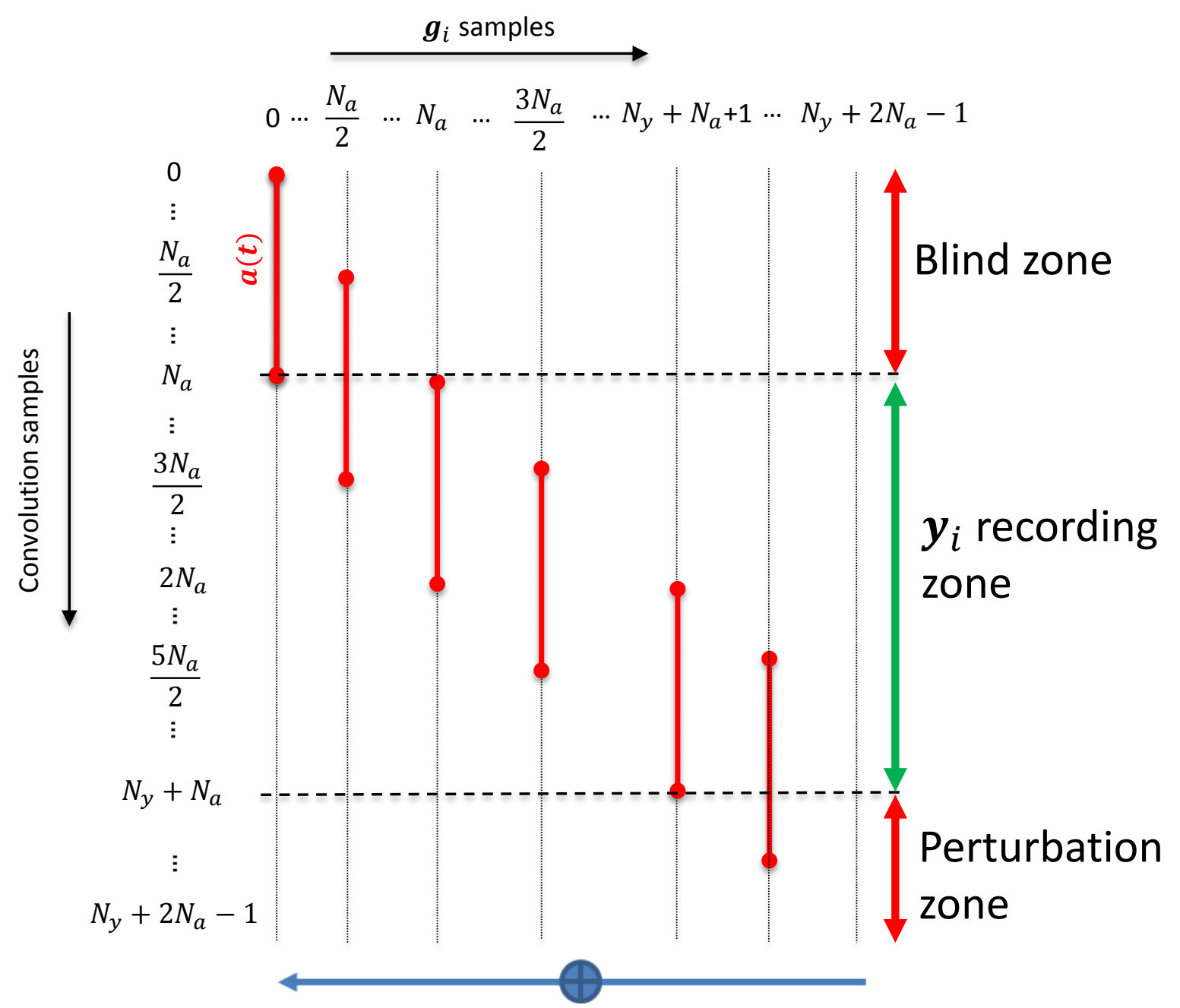

A is a $N_{y}$ by $N_{y}+N_{a}-1$ matrix 
Emission/reception of $N_{p w i}$ plane waves carrying signals $a^{k}(t)$

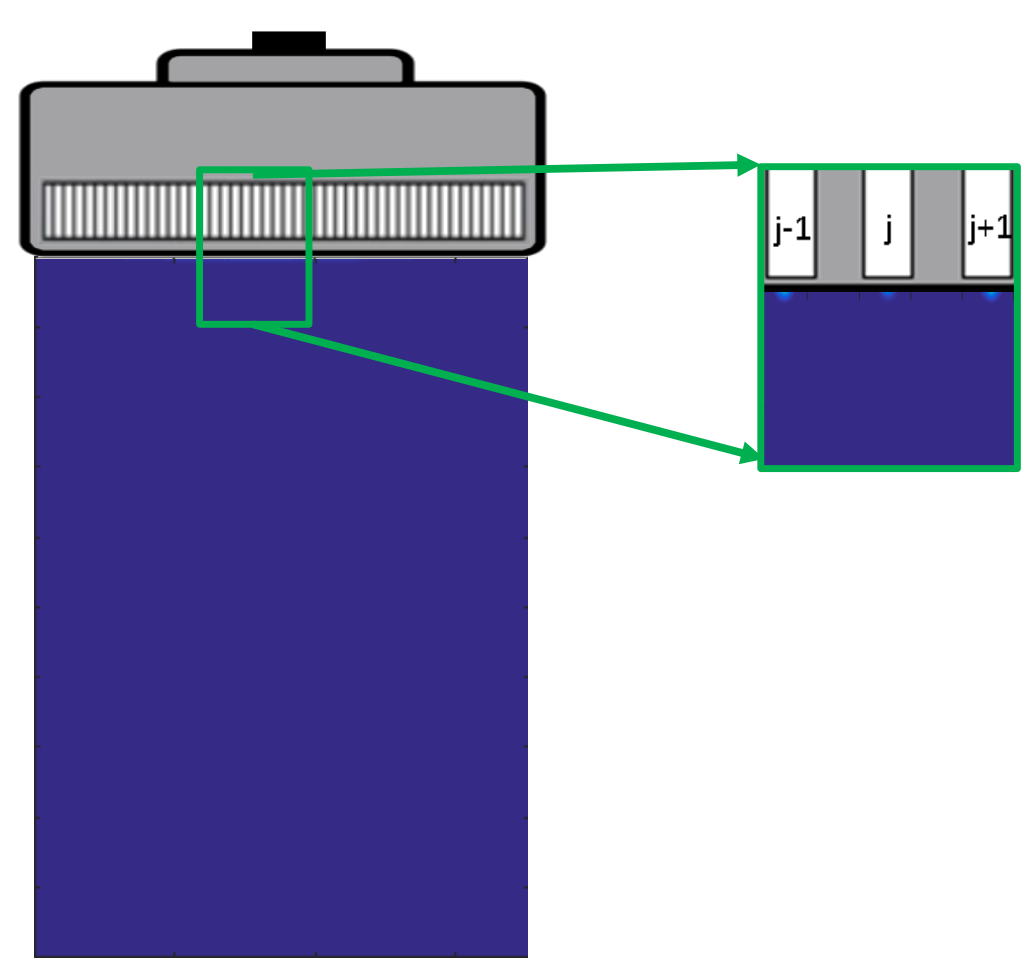

$$
a_{j}(t)=\sum_{k=1}^{N_{p w i}} a^{k}(t) * \delta\left(t-t_{j}^{k}\right) \quad k-\text { plane wave index }
$$$$
y_{i}(t)=\sum_{k=1}^{N_{p w i}} a^{k}(t) * g_{i}^{k}(t)+v_{i}(t)
$$$$
\times \stackrel{\oplus 11}{\downarrow_{N_{p w i}}}
$$

$$
\begin{aligned}
\mathbf{y}_{i}=\sum_{k=1}^{p \mathbf{A}^{k} \times \mathbf{g}_{i}^{k}+} \mathbf{v}_{i} & \\
\text { Replacing: } & \left\{\begin{array}{lll}
\mathbf{A}_{c}=\left[\begin{array}{llll}
\mathbf{A}^{1} & \mathbf{A}^{2} & \mathbf{A}^{3} \cdots & \mathbf{A}^{N_{p w i}}
\end{array}\right] \\
\mathbf{g}_{i}=\left[\begin{array}{llll}
\mathbf{g}_{i}^{1} & \mathbf{g}_{i}^{2} & \mathbf{g}_{i}^{3} \cdots & \mathbf{g}_{i}^{N_{p w i}}
\end{array}\right]
\end{array}\right.
\end{aligned}
$$

$$
\mathbf{y}_{i}=\mathbf{A}_{c} \overline{\mathbf{g}}_{i}+\mathbf{v}_{i}
$$

Estimate $\overline{g_{i}}(t)$ to reconstruct the ultrasound images

For this paper we used Linear Square Estimator: $\quad \hat{\mathbf{g}}_{i}=\left(\mathbf{A}_{c}^{T} \mathbf{A}_{c}\right)^{-1} \mathbf{A}_{c}^{T} \mathbf{y}_{i}$ 
Constraints for a well-posed system

$$
\mathbf{A}_{c}=\left[\begin{array}{llll}
\mathbf{A}^{1} & \mathbf{A}^{2} & \mathbf{A}^{3} \cdots & \mathbf{A}^{N_{p w i}}
\end{array}\right]
$$

$A_{C}$ size $: N_{y}$ rows, $N_{p w i}\left(N_{y}+N_{a}-1\right)$ columns

Constraint 1 , on the medium:

$$
g_{i}[z]=0, \forall z \in\left[0 \ldots N_{a}\right] \cup\left[N_{y}+N_{a}-1 \ldots N_{y}+2 N_{a}-1\right]
$$

New $A_{C}$ size: $N_{y}$ rows, $N_{p w i} \times N_{g}$ columns

$$
\text { with: } N_{y}=N_{a}+N_{g}-1
$$

Constraint 2, on the length of $a^{k}(t)$ :

$$
N_{a}=\left(N_{p w i}-1\right) N_{g}+1
$$

The emitted signals $a^{k}$ must be $N_{p w i}-1$ times longer than the impulse response of the medium

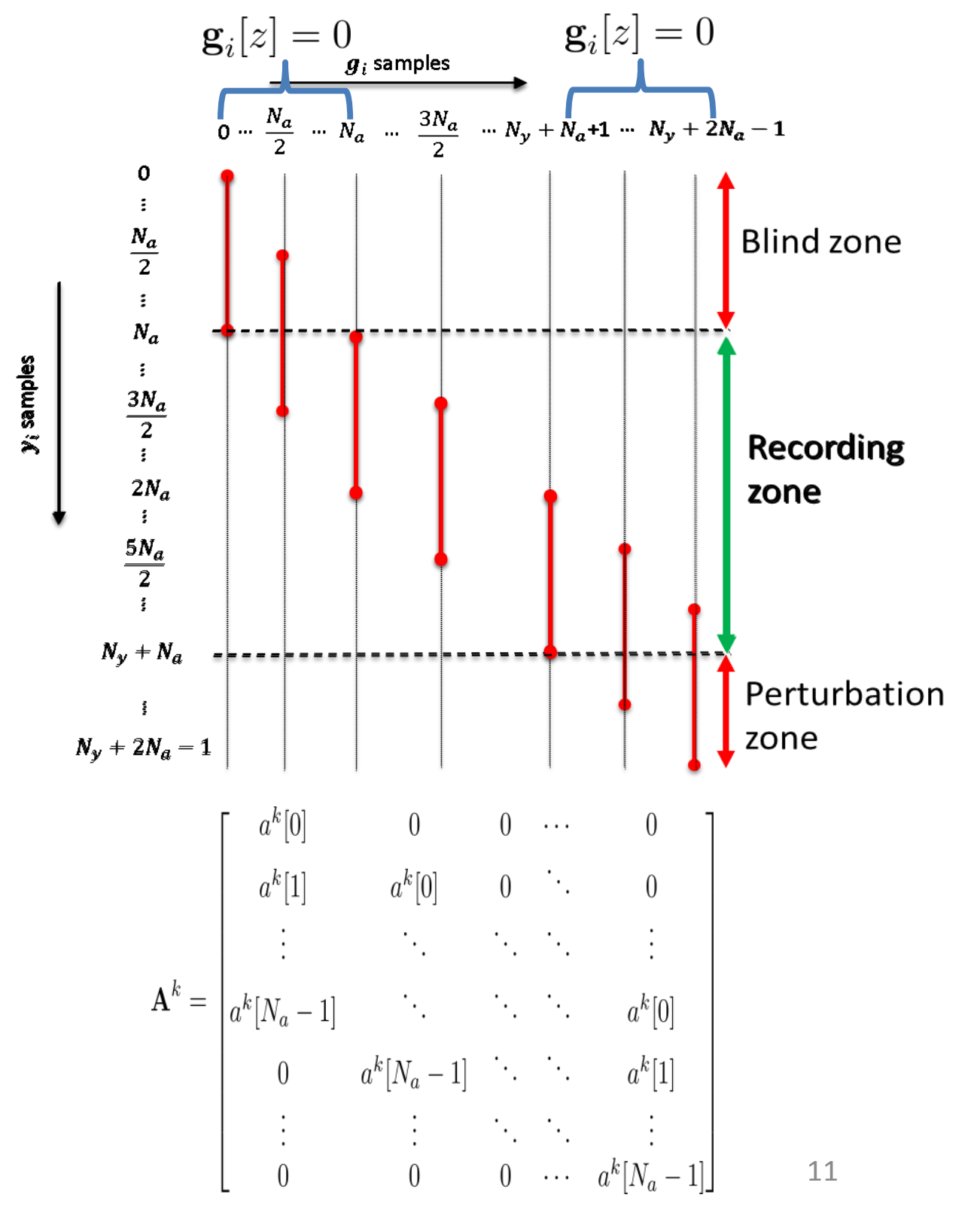


Constraints on the correlation of the emitted signals

$$
\mathbf{A}_{c}=\left[\begin{array}{ll}
\mathbf{A}^{1} & \mathbf{A}^{2}
\end{array}\right], N_{y} \text { rows by } 2 N_{g} \text { column matrix }
$$

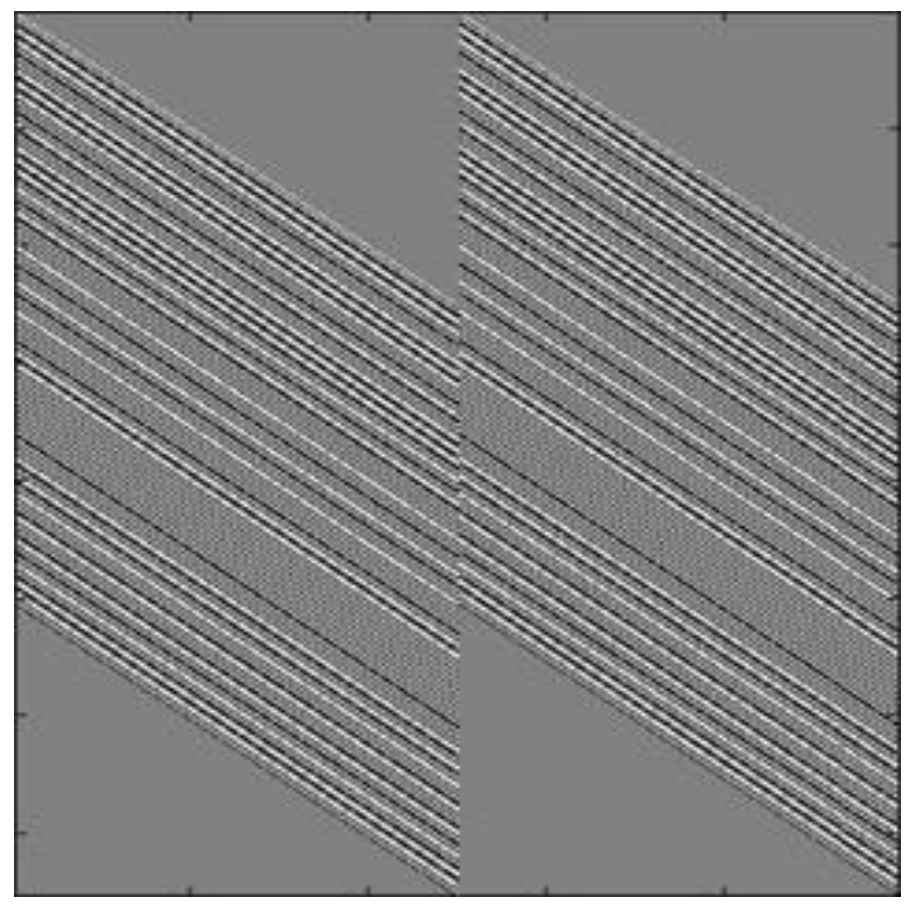

Low mutual coherence of $A_{c}=>A_{c}$ well conditionned

$$
\text { Excitation signals: } a^{k}(t)=s^{k}(t) \sin \left(2 \pi f_{0} t\right)
$$

$$
s^{k}(t) \text { is a pseudo-orthogonal code }
$$

\section{Frequency shift to $f_{0}$ using BPSK modulation}


Constraints on the correlation of the emitted signals

$\mathbf{A}_{c}=\left[\begin{array}{ll}\mathbf{A}^{1} & \mathbf{A}^{2}\end{array}\right], N_{y}$ rows by $2 N_{g}$ column matrix

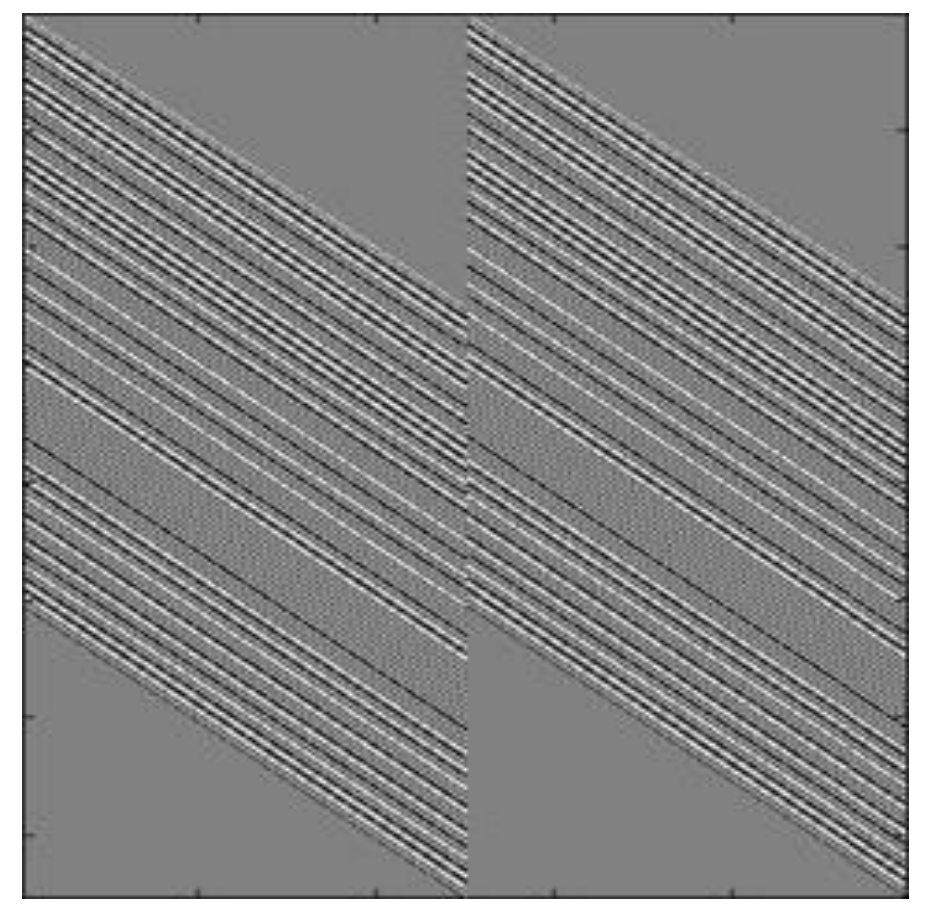

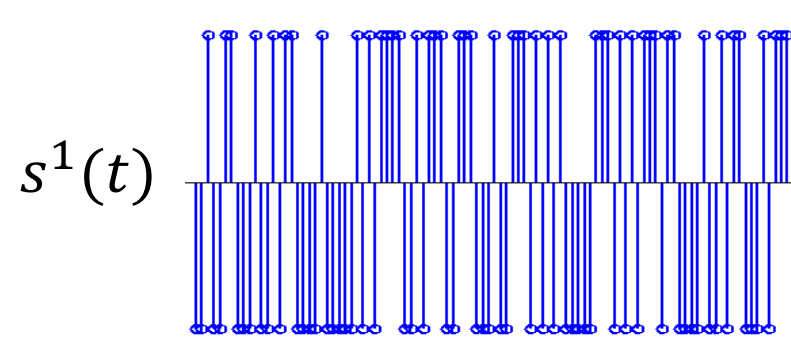

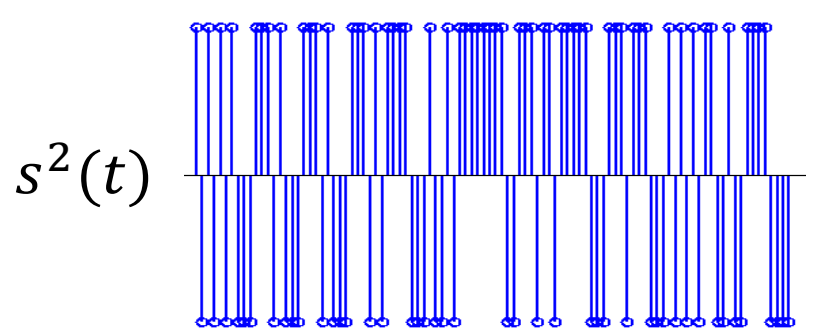

$a^{1}(t)$

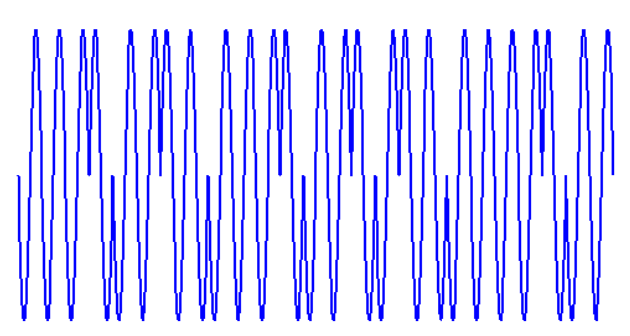

$a^{2}(t)$

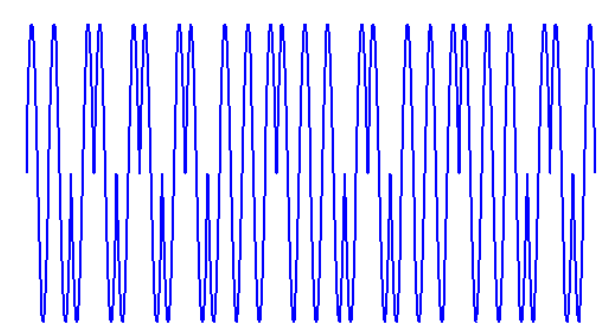

$$
a^{1}(t) \text { auto-corr. }
$$

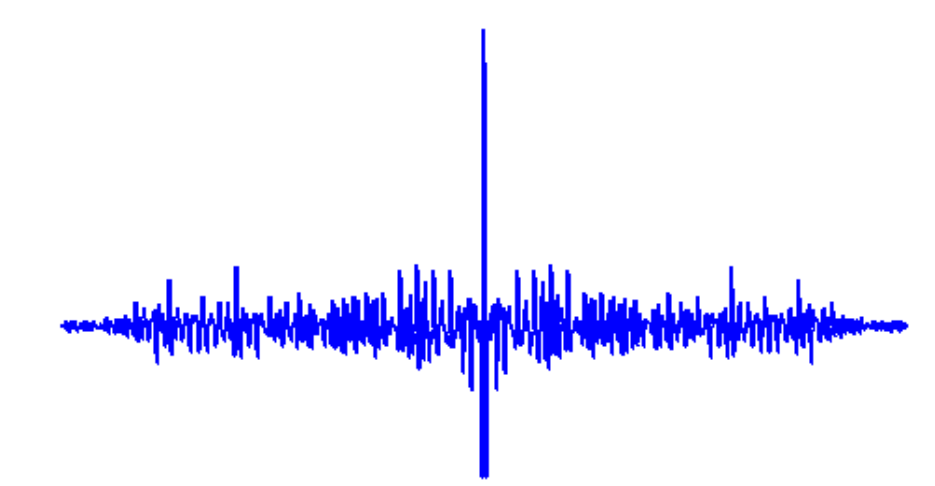

-

$a^{1}(t), a^{2}(t)$ cross-corr. 


\section{Field II $\left.\right|_{[1][2]}$ simulations results}

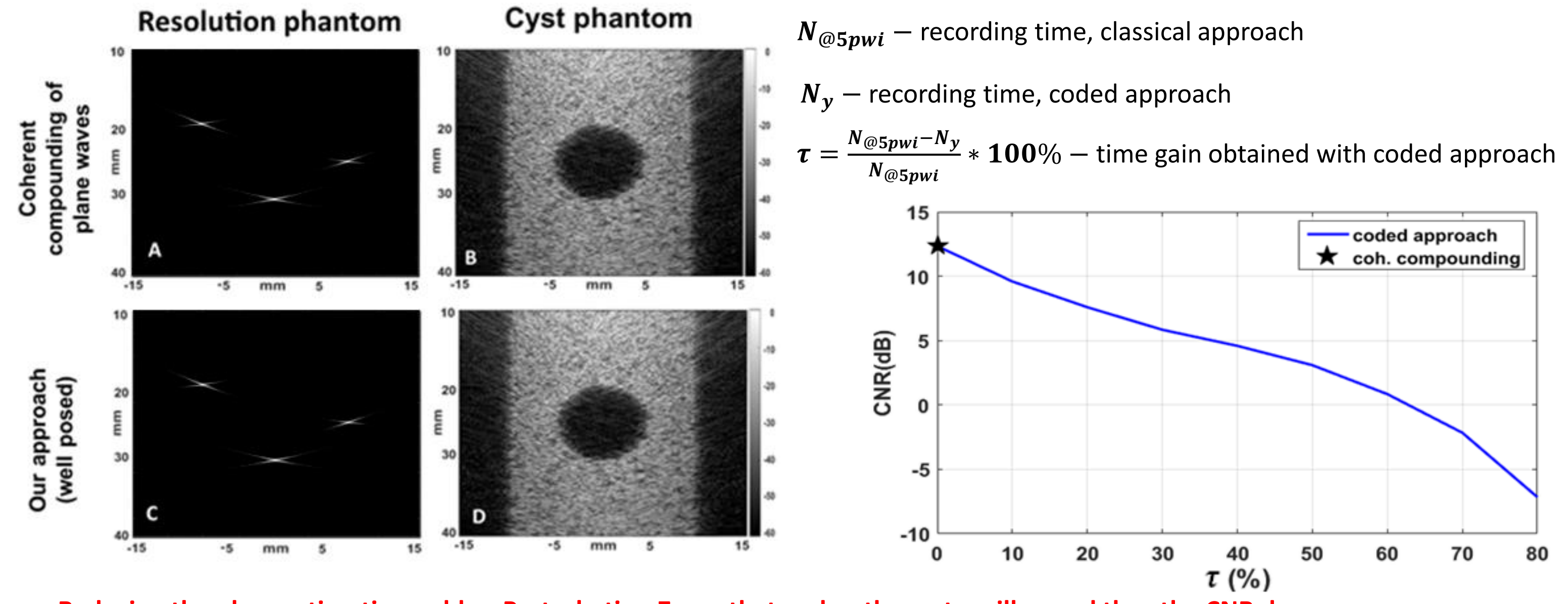

Reducing the observation time adds a Perturbation Zone, that makes the system ill-posed thus the CNR drops

[1] J. A. Jensen, MBEC, 1996

[2] J. A. Jensen et al., TUFFC, 1992 


\section{Conclusion}

A mathematical model of the simultaneous coded plane wave imaging

The physical constraints on the medium and emitted signals for a well posed system

Validation of the imaging process model without time gain

Increasing the frame rate leads to image quality decrease

Q: What's next?

Implement regularization to solve the inverse problem 
